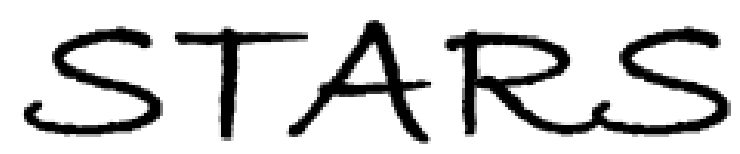

University of Central Florida

STARS

Faculty Bibliography 2000s

Faculty Bibliography

$1-1-2000$

\title{
Crossover behavior in dynamics of frozen ferrofluids
}

Xiaodong Duan

University of Central Florida

Jinlong Zhang

University of Central Florida

Weili Luo

University of Central Florida

Find similar works at: https://stars.library.ucf.edu/facultybib2000 University of Central Florida Libraries http://library.ucf.edu

This Article; Proceedings Paper is brought to you for free and open access by the Faculty Bibliography at STARS. It has been accepted for inclusion in Faculty Bibliography 2000s by an authorized administrator of STARS. For more information, please contact STARS@ucf.edu.

\section{Recommended Citation}

Duan, Xiaodong; Zhang, Jinlong; and Luo, Weili, "Crossover behavior in dynamics of frozen ferrofluids" (2000). Faculty Bibliography 2000s. 2509.

https://stars.library.ucf.edu/facultybib2000/2509

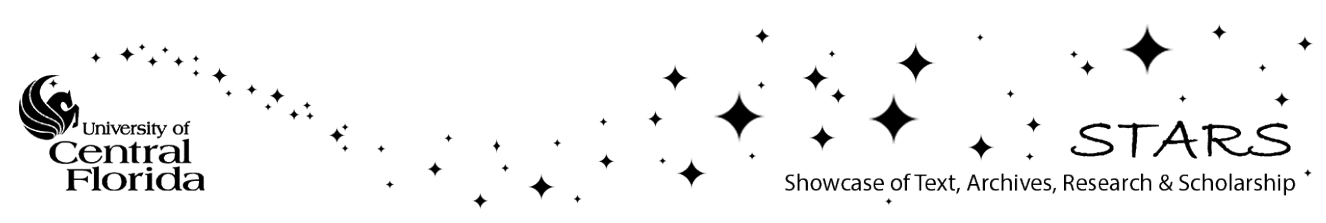




\section{Crossover behavior in dynamics of frozen ferrofluids}

Cite as: Journal of Applied Physics 87, 6935 (2000); https://doi.org/10.1063/1.372891

Published Online: 28 April 2000

Xiaodong Duan, Jinlong Zhang, and Weili Luo

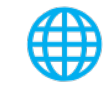

\section{Instruments for Advanced Science}

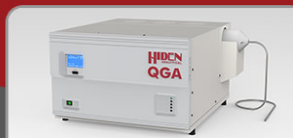

Gas Analysis

- dynamic measurement of reaction gas streams

, catalysis and thermal analysis

- dissohed species probes
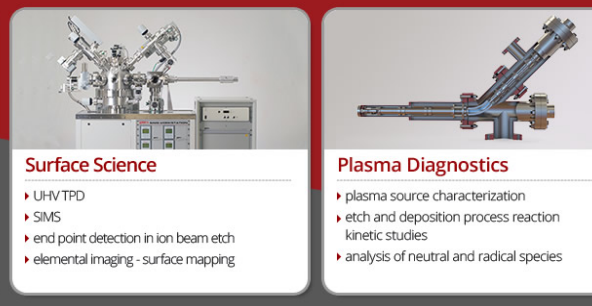

Plasma Diagnostics

- plasma source characterization

tch and deposition process reaction

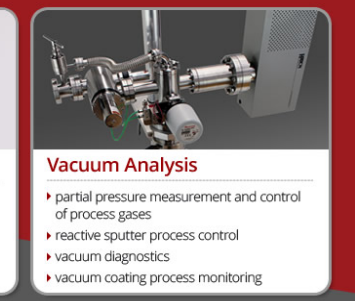




\title{
Crossover behavior in dynamics of frozen ferrofluids
}

\author{
Xiaodong Duan, Jinlong Zhang, and Weili Luo ${ }^{\text {a) }}$ \\ Department of Physics, University of Central Florida, Orlando, Florida 32816
}

The imaginary part of susceptibility, $\chi^{\prime \prime}$, in frozen ferrofluids was measured as functions of temperature, frequency, and concentration of magnetic particles. In a very dilute region, where magnetic interaction is negligible, the relaxation time is mainly determined by Neel's relaxation in isolated particles and the frequency-dependent peak temperature in $\chi^{\prime \prime}(T), T_{p}$, obeys the Arrhenius law. In the moderate concentration range, $T_{p}$ has a Vogel-Fulcher (VF) relaxation with the measuring frequency. However, for concentration larger than a certain value, deviation from VF law occurs. Simulations show that the VF relation could be attributed to Ising spin-glass-like random and frustrated interaction between magnetic moments of particles. We suggest that particle configuration before freezing is critical to determine the distribution of pair exchange parameters. For high concentration, there are more ferromagnetic than antiferromagnetic bonds. The deviation from VF law at high concentration could be due to short range correlation among spins. (c) 2000 American Institute of Physics. [S0021-8979(00)24908-6]

The relaxation behavior in frozen ferrofluids has been recently investigated as a disordered system with competing interactions. ${ }^{1-8}$ Recently, some results indicate the existence of a spin-glass-like behavior in frozen ferrofluids ${ }^{4,6}$ These papers have shown a critical slowing down for the average relaxation time and a divergence of the nonlinear susceptibility at finite temperature $T_{g} .{ }^{4,5}$ It is also found that the frequency-dependent peak temperatures in the imaginary susceptibility in ferrofluids obey Vogel-Fulcher law. ${ }^{3}$ The aging phenomenon was found at low temperature and it confirmed the existence of glass or spin glass phase. ${ }^{6,7}$ Therefore, it is now accepted that there is cooperative behavior in frozen ferrofluids. However, the relaxation behavior depends on magnetic particle concentration and so far most of work mentioned only study one or a small range of concentration. In this article, we experimentally study the dynamic susceptibility for concentration from extreme dilute limit $(0.0045 \%)$ to concentrated regime $(13 \%)$. We found that the peak temperature versus measuring frequency can be described by Arrhenius law in a very dilute limit, crossing over to Vogel-Fulcher law at more concentrated regime, and finally deviating from VF relation at highest concentration. Comparing with the simulation results suggests that these crossover behaviors are associated with single moment relaxation, cooperated relaxation from random, frustrated interaction, to the one originated from nonfrustrated interaction. Since the frozen ferrofluid could be used as a model system to study the effects of dipolar interaction on relaxation, a complete study of such a system is necessary and could shed a light on other disordered systems such as glass-forming liquids, ${ }^{9}$ spin-glasses,${ }^{10}$ polymers, ${ }^{11}$ dielectric relaxors, ${ }^{12}$ and others.

Experiments were performed on ferrofluid samples consisting of magnetic particles dispersed in alkylnaphthalenes, abbreviated as ABF. Each particle has a mean diameter of 7 $\mathrm{nm}$. Particles are coated with surfactant to avoid agglomera-

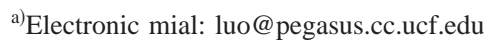

tion. Particles interact with each other through the long range dipole force. The sample was sealed inside a small quartz tube. The ac susceptibility measurements were performed with a SQUID ac susceptometer. The samples with very dilute concentration $(0.0045 \%$, named ABFL), moderate concentration (10.4\%, named ABFM), and high concentration $(12.9 \%$, named $\mathrm{ABFH})$ are used in the experiments. Each sample was first rapidly cooled down from room temperature to $4.5 \mathrm{~K}$ in zero field. The cooling rate was approximately $100 \mathrm{~K} / \mathrm{min}$. Then, the susceptibility $\chi^{\prime \prime}$ was measured at temperature $T$ in intervals of $4 \mathrm{~K}$ from 6 to $300 \mathrm{~K}$ for five different frequencies $f=0.1,1,10,100$, and $1000 \mathrm{~Hz}$, respectively. The field amplitude was 1 Oe except for sample ABFL, where the amplitude was 5 Oe because of weak signal. The linear relation between the magnetization and the field was confirmed in this field region. In Fig. 1(a), the imaginary part of ac susceptibility, $\chi^{\prime \prime}$, is plotted as function of temperature, $T$, at different frequencies for ABFM. In Fig. $1(\mathrm{~b})$, the peak temperature in $\chi^{\prime \prime}(T)$ were plotted as a function of $1 /\left(\ln f-\ln f_{0}\right)$, where $1 / f_{0}$ is $10^{-9} \mathrm{~s}$ for all concentration (ABFL, ABFM, and $\mathrm{ABFH}$ ). The straight line represents the Vogel-Fulcher law: $f=f_{0} \exp \left[A / k\left(T-T_{0}\right)\right]$. In order to understand the experiment results, we perform simulation in the following paragraph.

In a very dilute region, we can neglect dipole-dipole interaction between particles. The relaxation originates from Neel mechanism, following activated Arrhenius law: $\tau=\tau_{0} \exp \left(K v / k_{B} T\right)$, where $K$ is the anisotropy energy coefficient, $v$ the volume of a particle. The general expression of $\chi^{\prime \prime}$ can be obtained from fluctuation and dissipation theory, ${ }^{13}$

$$
\chi^{\prime \prime}(\omega, T)=\frac{M_{s}^{2} v}{3 k_{B} T} \int_{0}^{\infty} \frac{g(\tau, T) \omega \tau}{1+(\omega \tau)^{2}} d \tau,
$$

$M_{s}$ is the saturation magnetization. The $1 / 3$ factor comes from averaging the random angle between a dipole and the field directions. $g(\tau, T)$ is the distribution function for relaxation time. For noninteracting system, $g(\tau, T)$ could be due 

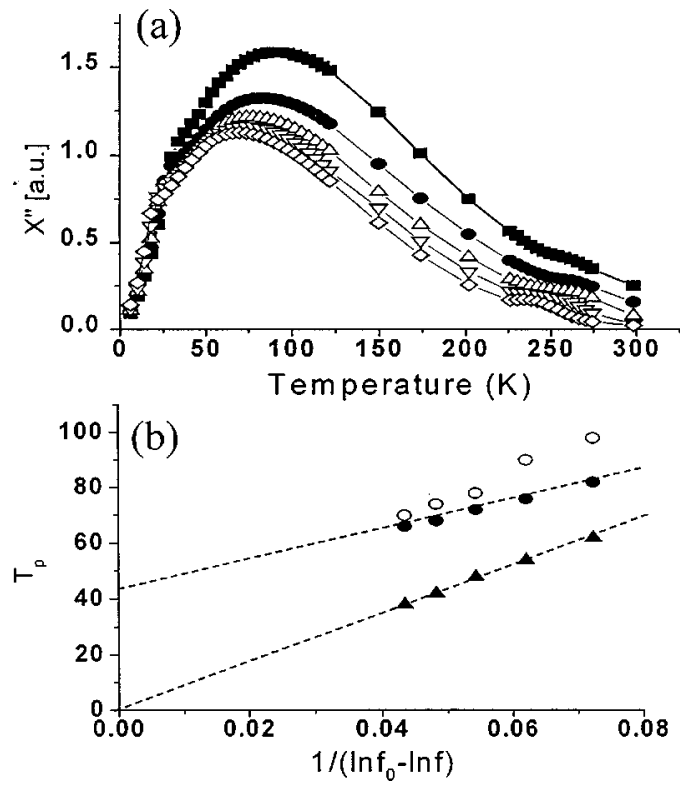

FIG. 1. (a) Imaginary part of susceptibility, $\chi^{\prime \prime}$, for ABFM vs temperature for following measurement frequencies: $0.1 \mathrm{~Hz}(\bigcirc), 1 \mathrm{~Hz}(\bullet), 10 \mathrm{~Hz}(\boldsymbol{\square})$, $100 \mathrm{~Hz}(\square)$, and $1000 \mathrm{~Hz}(\triangle)$. (b) Experimental results for peak temperatures, $T_{p}$ in $\chi^{\prime \prime}(T)$ vs $1 /\left(\ln f-\ln f_{0}\right)$ for $\operatorname{ABFL}(\mathbf{\Lambda}), \operatorname{ABFM}(\bullet)$, and $\mathrm{ABFH}$ (O).

to the size distribution of particles. If all the particles have the same volume, Eq. (1) can be simplified as

$$
\chi^{\prime \prime}(\omega, T)=\frac{M_{s}^{2} v}{3 k_{B} T} \frac{\omega \tau_{0} \exp \left(K v / k_{B} T\right)}{\left[\omega \tau_{0} \exp \left(K v / k_{B} T\right)\right]^{2}+1} .
$$

Considering the size distribution effect, one can assume that $M_{s}$ is proportional to the particle volume, then $\chi^{\prime \prime}$ can be written as

$\chi^{\prime \prime}(\omega, T)=\frac{\bar{M}_{s}^{2}}{3 k_{B} T \bar{v}} \int_{0}^{\infty} \frac{\omega \tau_{0} \exp \left(K v / k_{B} T\right)}{\left[\omega \tau_{0} \exp \left(K v / k_{B} T\right)\right]^{2}+1} v^{2} f(v) d v$,

where $\bar{M}_{s}, \bar{v}$ are average values. $f(v)$ is the distribution function for particle volume. $f(v)$ is often represented by the log-normal function. ${ }^{14}$ In moderate concentration region, the particles are still randomly distributed. However, the dipoledipole interaction becomes important. Because of the barrier due to the anisotropy energy, the magnetic moment tends to align with the easy axis within the particle and can be treated as a point dipole. The interaction between two dipole moments $m_{1}$ and $m_{2}$ is

$$
\hat{J}_{i j}=-\frac{3 \cos \varphi_{1} \cos \varphi_{2}-\cos \left(\varphi_{1}-\varphi_{2}\right)}{\mathbf{r}_{12}^{3}} m_{1} m_{2},
$$

where $\mathbf{r}_{12}$ is the distance between $m_{1}$ and $m_{2}, \varphi_{1}$ and $\varphi_{2}$ are the angles between $m_{1}, m_{2}$, and the vector from $m_{1}$ to $m_{2}$, respectively. In order to make a clear discussion, the magnitude and direction of $m_{i}$ are separated by introducing a quantity $S_{I}$. When the projection of dipole to the external field is positive, $S_{I}$ takes +1 , otherwise, $S_{I}$ takes -1 . An effective exchange parameter $J_{i j}$ is introduced. So the model Hamiltonian is $H_{\text {mod }}=-\sum_{i \neq j} J_{i j} S_{i} S_{i}$. The effective exchange parameter $J_{i j}$ is determined by the random direction and the

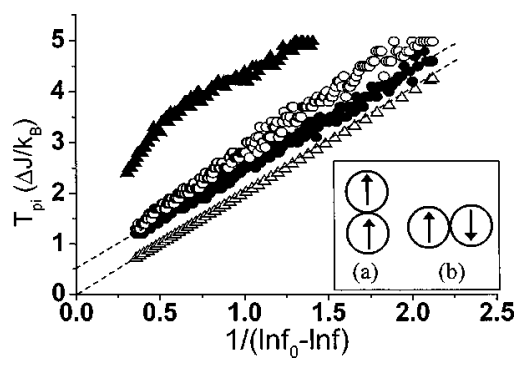

FIG. 2. Results from MC simulation: peak temperatures in $\chi^{\prime \prime}(T)$ vs the $1 /\left(\ln f-\ln f_{0}\right)$ for the dilute $(\triangle)$, moderate $(\boldsymbol{O})$, and concentrated $(\bigcirc$ and $\mathbf{\Delta})$ samples. The temperature is normalized by the distribution width $\Delta J$ in (8). In the simulation for concentrated samples, we assumed that $J_{0}=0.5 \Delta J(\bigcirc)$ and $J_{0}=\Delta J(\mathbf{\Delta})$ in the drift Gaussian distribution. For simulation of dilute concentration, the energy barrier is assumed $E=2 \Delta J$. Insert: the easy magnetization axis of two particles are in alignment. $J_{i j}>0$, suggesting ferromagnetic bond in model Hamiltonian. The attractive energy is $E_{f}$ $=-2 \mu_{0} M_{s}^{2} / d^{2}$, where $d$ is the particle diameter. (b) The easy magnetization axes of two particles are in parallel. $J_{i j}<0$ indicating antiferromagnetic bond in the model Hamiltonian. The attractive energy is $E_{a f}$ $=-\mu_{0} M_{s}^{2} / d^{2},\left|E_{a f}\right|<\left|E_{f}\right|$.

distance of magnetic dipoles, the distribution of $J_{i j}$ could be approximated by a Gaussian $P\left(J_{i j}\right) \propto \exp \left[-J_{i j}^{2} / 2(\Delta J)^{2}\right]$. This is the standard model or Ising spin glass. The imaginary part of the susceptibility can be calculated by Eq. (2), where the relaxation time distribution function $g(\tau, T)$ was found by Monte Carlo (MC) simulation, using the above model Hamiltonian. ${ }^{8}$ For a polydispersed system, both dipole interaction and the size distribution contribute to $g(\tau, T)$. We noticed that the intrinsic relaxation time is different for particles with different size. Generally, two magnetic particles with different intrinsic time clock could not synchronize if the dipole-dipole interaction energy is smaller than the energy barrier. In that case, the interaction for long time average will be zero for two particles with different sizes. So we can approximately write down the relaxation time distribution function as $g(\tau, T)=g_{i}(\tau, T) g_{v}(\tau, T)$, where $g_{i}$ is the distribution function for the dipole interaction and $g_{v}$ for the particle size distribution. The imaginary susceptibility for polydispersed frozen ferrofluid can be expressed as

$$
\begin{aligned}
\chi^{\prime \prime}(\omega, T)= & \frac{\bar{M}_{s}^{2}}{3 k_{B} T \bar{v}} \int_{0}^{\infty} v^{2} f(v) d v \\
& \times \int_{0}^{\infty} \frac{\omega \tau \exp \left(K v / k_{B} T\right) g_{I}(\tau, T)}{1+\left[\omega \tau \exp \left(K v / k_{B} T\right)\right]^{2}} d \tau .
\end{aligned}
$$

$g_{i}$ could be obtained from MC simulation. ${ }^{8}$ In a high concentration region, the dipole-dipole interaction becomes even stronger and can affect the particle configuration in liquid state. When the solvent is frozen, the configuration is frozen as well. We compare two situations shown in the insert of Fig. 2. When the easy magnetization axes of two particles are in alignment [Fig. 2, inset (a)], the exchange parameter $J_{i j}$ is greater than zero, representing ferromagnetic bond. While for parallel easy axes that are not in alignment [Fig. 2, inset (b)], $J_{i j}$ is less than zero, representing the antiferromagnetic bond. Due to the nature of dipole-dipole interaction, the configuration in Fig. 2 [inset (a)] has lower free 
energy than the one in Fig. 2 [inset (b)]. So the system will favor the ferromagnetic bond at high concentration. A simple model for the high concentration frozen ferrofluid can be expressed as $H_{\text {mod el }}=-\sum_{i \neq j} J_{i j} S_{i} S_{i}$ and the distribution of the exchange parameter $P\left(J_{i j}\right) \propto \exp \left[-\left(J_{i j}-J_{0}\right)^{2} / 2(\Delta J)^{2}\right]$ with $J_{0}>0$. The imaginary susceptibility can be calculated by Eq. (1) or (5) except that $g(\tau, T)$ is obtained from MC simulation for the model Hamiltonian in which the distribution of exchange interaction follows a shifted Gaussian.

The simulation results for the peak temperatures, $T_{p}$, in the imaginary part of the susceptibility, $\chi^{\prime \prime}(T, f)$, are shown in Fig. 2, where $f$ is the measuring frequency of simulation and $1 / f_{0}$ is the $\tau_{0}$, the unit spin-flip time used in MC simulation. In order to simplify the calculation, we only consider the monodispersed system. As we can see from Fig. 2, the simulation results qualitatively agreed with experiments very well. For the dilute limit, the extrapolated line goes through the origin, suggesting $T_{0}=0$, then Arrhenius law is recovered as expected since relaxation for single particle should exhibit activated behavior described by Neel's relaxation. At the moderate concentration, VF law was found, the finite diverse temperature, $T_{0}$, arose from the interaction between particles. At high concentration, the behavior can no longer be described by any simple relation. However, the simulation results suggest that the deviation from VF law comes from the change of particle configuration. In moderate concentration, the particle distribution is still completely random. But in high concentration, the interaction energy between particles in the liquid state can be comparable to or larger than the thermal energy and thus changes the particle distribution. As a result, there were more ferromagnetic than antiferromagnetic type interactions when $\mathrm{ABFH}$ was frozen. We have measured the kerosene based ferrofluid $(\mathrm{KBF})^{2,8}$ and the deviation from VF law occurred at concentration of $6 \%-8 \%$ compare to the $10 \%-12 \%$ of the ABF sample. The frozen temperature of kerosene is at $151 \mathrm{~K}$ which is lower than that of alkylnaphthalenes, $263 \mathrm{~K}$, it might be the reason of higher deviation concentration in $\mathrm{ABF}$.

In conclusion, the dynamic susceptibility was studied for $\mathrm{ABF}$ with different concentrations. We found that in a dilute region, the relaxation time are mainly determined by Neel's relaxation in the isolated particles. In the moderate and high concentration ranges, the relaxation behavior not only depends on the strength of the pair interaction but also depends on the distribution of particles. Simulation showed that the VF relation could be attributed to Ising spin-glass-like interaction where the distribution function for exchange parameters is a Gaussian centered at zero. Shifting the center of the Gaussian from zero to a nonzero value leads to the deviation from VF law at high concentration, suggesting correlation in the system.

The authors are grateful to Dr. S. Taketomi for providing samples. This work is supported by the National Science Foundation Young Investigator Award (W.L.).

${ }^{1}$ W. Luo et al., Phys. Rev. Lett. 67, 2721 (1991).

${ }^{2}$ T. Jonson et al., Phys. Rev. Lett. 75, 4138 (1995).

${ }^{3}$ J. Zhang, C. Boyed, and W. Luo, Phys. Rev. Lett. 77, 390 (1996).

${ }^{4}$ C. Djurberg et al., Phys. Rev. Lett. 79, 5154 (1997).

${ }^{5}$ H. Mamiya, I. Nakatani, and T. Furubnayashi, Phys. Rev. Lett. 80, 177 (1998).

${ }^{6}$ T. Jonsson et al., Phys. Rev. Lett. 81, 3976 (1998).

${ }^{7}$ H. Mamiya, I. Nakatani, and T. Furubayashi, Phys. Rev. Lett. 82, 4332 (1999).

${ }^{8}$ X. Duan, J. Zhang, and W. Luo (unpublished).

${ }^{9}$ M. D. Ediger, C. A. Angell, and S. Nagel, J. Phys. Chem. 100, 13200 (1996).

${ }^{10}$ See, Spin Glasses and Random Fields, edited by A. P. Young (World Scientific, Singapore, 1998).

${ }^{11}$ C. Angell, Science 267, 1924 (1995).

${ }^{12}$ D. Viehland, S. J. Jang, and L. E. Cross, J. Appl. Phys. 69, 414 (1991).

${ }^{13} \mathrm{~S}$. Dattagupta, Relaxation Phenomena in Condensed Matter Physics (Academic, London, 1987).

${ }^{14}$ T. Jonsson, J. Mattsson, P. Nordblad, and P. Svedlindh, J. Magn. Magn. Mater. 168, 269 (1997). 